\title{
Lightweight Design of a Rear Axle Connection Bracket for a Heavy Commercial Vehicle by Using Topology Optimisation: A Case Study
}

\author{
Mehmet Murat TOPAÇ*, Merve KARACA**, Birkan AKSOY***, Uğur DERYAL****, \\ Levent BILAL $* * * * *$ \\ *Faculty of Engineering, Dokuz Eylül University, 35397 Izmir, Turkey, E-mail: murat.topac@deu.edu.tr \\ **The Graduate School of Natural and Applied Sciences, Dokuz Eylül University, 35397 Izmir, Turkey, \\ E-mail: merve.karaca@ogr.deu.edu.tr \\ ***Faculty of Engineering, Dokuz Eylül University, 35397 Izmir, Turkey,E-mail: birkan.aksoy@ogr.deu.edu.tr \\ ****BMC Otomotiv Sanayi ve Ticaret A.Ş., Pınarbaşı, 35060 Izmir, Turkey,Turkey, E-mail: ugur.deryal@ogr.deu.edu.tr \\ ******Faculty of Engineering, Dokuz Eylül University, 35397 Izmir, Turkey, E-mail: levent.bilal@ogr.deu.edu.tr \\ cross $^{\text {ref }}$ http://dx.doi.org/10.5755/j01.mech.26.1.23141
}

\section{Introduction}

Four-link solid axles are commonly being used in articulated heavy commercial trucks due to their simplicities. In this design, the connection between the rear axle and chassis is ensured by two couples of control arms [1]. Fig. 1, b illustrates a detailed design of the system [2]. Here, each lower control arm 7 is attached to the chassis 4 by means of a connection bracket 6 as shown in Fig. 2. Because of their critical function, these connection brackets should satisfy the strength conditions during the service as well as the sufficient rigidity. On the other hand, due to the economic concerns, vehicle components should be designed in lightest possible way. Design examples of lightweight connection brackets are given in Fig. 2.

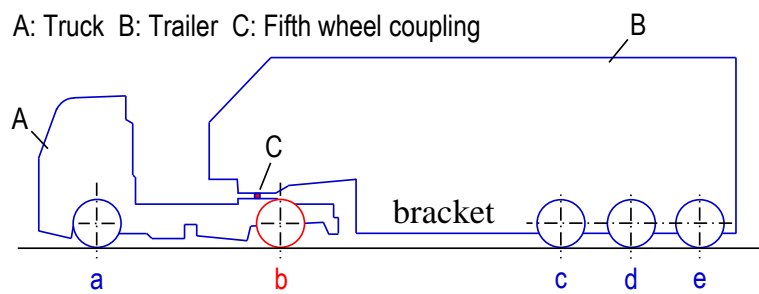

a

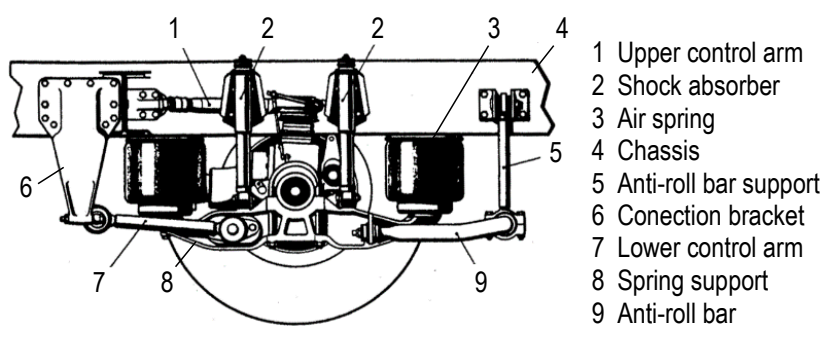

b

Fig. 1 Schematic for the articulated heavy commercial vehicle: a - structure of the four-link axle (axle "b"); $\mathrm{b}-[2]$

In this paper, mechanical design stages of a connection bracket which is used in the rear axle of an articulated heavy commercial truck were summarised. In the scope of the design process, a combination of various techniques namely, multibody dynamics (MBD), finite element (FE) analysis topology optimisation and response surface methodology (RSM)-based design optimisation were employed. Design study consists of two major steps, namely, topology optimisation-based mass reduction and response surface methodology-based stress reduction. In order to determine the design load, a detailed MBD model of the articulated truck was utilised.
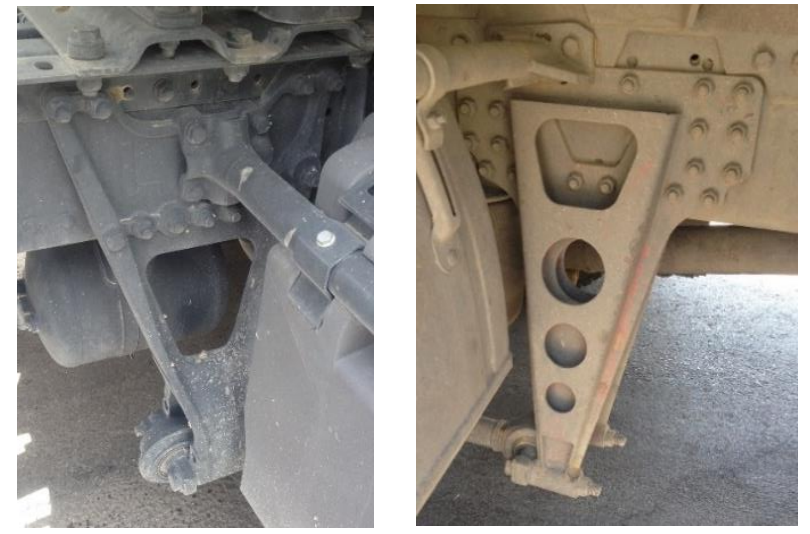

Fig. 2 Design examples for the lightweight connection

\section{Literature review}

In topology optimisation field, several studies have been achieved so far, whose scope covers theoretical developments as well as the applications of the method. One of the first topology optimisation approaches is the homogenisation method, which was developed by Bendsøe and Kikuchi in 1988 [3]. This method was followed by a material density formulation on a chassis design problem, which was studied by Jang and Chahande in 1995 [4]. In 1996, the homogenisation method was studied via the DYNA 3D software for an energy absorption problem by Mayer etal. [5]. Bendsøe and Sigmund applied the material density methodology on composite materials in 1999 [6]. They also summarised the material density method theory and the applications on mechanical problems in Topology Optimisation: Theory, Methods, and Applications in 2003 [7]. The level set method was offered as an alternative to the introduced topology optimisation methods by Zha and Haegawa in 1999 and Wang in 2003 [8-9]. Fredricson studied on the presence of joint elements in topology optimisation, which were not taken into account seriously earlier, and developed a methodology with the existence of joint elements in 2003 [10]. Jang et al. employed the method 
during the design of a flatbed trailer and examined the effects of topology optimisation on the torsional frequency and the total mass of the trailer in 2010 [11]. Similarly, the method was utilised for a truck frame design by Wang et al. in 2013 [12]. Tang et al. determined the loads that act on a suspension control arm by using multibody dynamics and accordingly employed the topology optimisation methodology for mechanical optimisation of control arms in 2014 [13]. This study aims to combine the multibody dynamics for the load determination according to the specific function of a connection bracket and a two-stage optimisation process. The first optimisation stage is to weight reduction, which employs topology optimisation in order to obtain optimal material distribution inside the total volume of the structure. After that, the initial design geometry composed. The second stage is design optimisation that concerns of achieving the optimum values of particular dimensions in order to accomplish minimum stress and deformation values.

\section{Material and method}

Topology optimisation is a mechanical design tool, which aims to employ the stiffest material distribution in a way that as light as possible. A great benefit of the method is its capability of generating individual designs in a given design volume, whose outline must not be defined by the designer. The working principle of the optimisation is removing material from less loaded volumes and retaining material on load-carrying regions, under the given circumstances. Accordingly, the finite elements are distinguished to solid elements or void elements. Therefore, the elements are re-defined with a pseudo-density value, which changes between zero and one that depends on the load-carrying condition. Homogenisation method is a popular topology optimisation approach based on computational FE methods, which was developed to utilise topology optimisation on isotropic materials and defines the pseudo-density as either zero or one. However, such sharp discrimination of pseudodensity results with a non-manufacturable structure with jagged edges and random holes inside the geometry. Another approach of topology optimisation is SIMP (Solid Isotropic Material with Penalisation) method which is based on a penalisation factor to enforce the intermediate densities to take values closer to either zero or one, in order to avoid the grey regions that compose of elements with intermediate densities. In this approach, the pseudo-density is defined as a continuous function, and is expressed as:

$$
0 \leq \rho_{\min } \leq \rho_{e} \leq 1
$$

Hence, the total structural volume is converted to:

$$
\int_{\Omega} \rho(x) d \Omega \leq V,
$$

here: $V$ is the volume of the initial structure. The global stiffness matrix should be written in terms of element pseudodensities. Therefore, it becomes:

$$
K(\rho)=\sum_{e=1^{1}}^{N E} \rho_{e}^{q} K_{e}, \quad q>1,
$$

where: $K_{e}$ is the stiffness of element e, $q$ is the penalisation factor to avoid intermediate densities, and the $K$ is the global stiffness matrix, which composes of the sum of elementary stiffness of each element. The value of penalisation factor $q$ is decided by taking the optimisation accuracy, mechanical properties of the material and the design variables into account. As it is revealed in [14], an expression of elasticity and pseudo-density is derived by means of the direct proportion between Young's modulus and the stiffness. Correspondingly, the elasticity tensor of element e can be written as:

$$
E_{e}\left(\rho_{e}\right)=E_{0} \rho_{e}^{q} .
$$

The elasticity of element e can also be expressed in terms of strain and stress of element $e$ :

$$
E_{e}=\frac{\sigma_{e}}{\varepsilon_{e}}
$$

The maximum stiffness is obtained when structural compliance is minimised. Thus, the minimum strain energy approach is employed to derive an expression of total compliance of geometry. The total compliance of a structure, which is subjected to load vector $f$ is given as:

$$
l(u)=\int_{\Omega} f u d \Omega
$$

According to the minimum strain energy approach, the optimisation problem can be written as:

$$
\begin{aligned}
& \min f^{T} u \text { s.t } E(e)=\rho(e)^{p} E_{0,}, \\
& \int_{\Omega} \rho(x) d \Omega \leq V ; \text { where } E_{e} \in E_{a d},
\end{aligned}
$$

here: $u$ and $E_{a d}$ denote the displacement vector and the admissible Young's modulus value, respectively. As a result of the minimisation of strain energy, which is subjected to the volume restriction constraint, the stiffest structure is obtained by the optimisation. However, the optimisation is mostly tended to result with a checkerboard-like microstructure, which is not only a non-manufacturable but also not safe against the stress concentrations. Therefore, a filtering process is subsequently applied to the structure, in order to eliminate the checkerboard problem. The stages of topology optimisation are summarised in Fig. 3.

\section{Load model}

\subsection{System description}

In many four-link rear axle applications, upper control arms are connected to the axle with an angle, $\sigma$. As a remarkable advantage of this arrangement, the links bear lateral forces that act on the axle during a turning motion. The kinematic scheme of four-link rear axle is given in Figs. 4 and 5. The schematic illustration apparently reveals that the lateral forces are carried by the upper control arms.

Consequently, it is understood that lateral forces and the moments related to these lateral forces are negligible 
on connection bracket either during linear motion due to the reaction forces of axle settlement, or during turning motion. Therefore, the lateral forces were not taken into account for the bracket load.

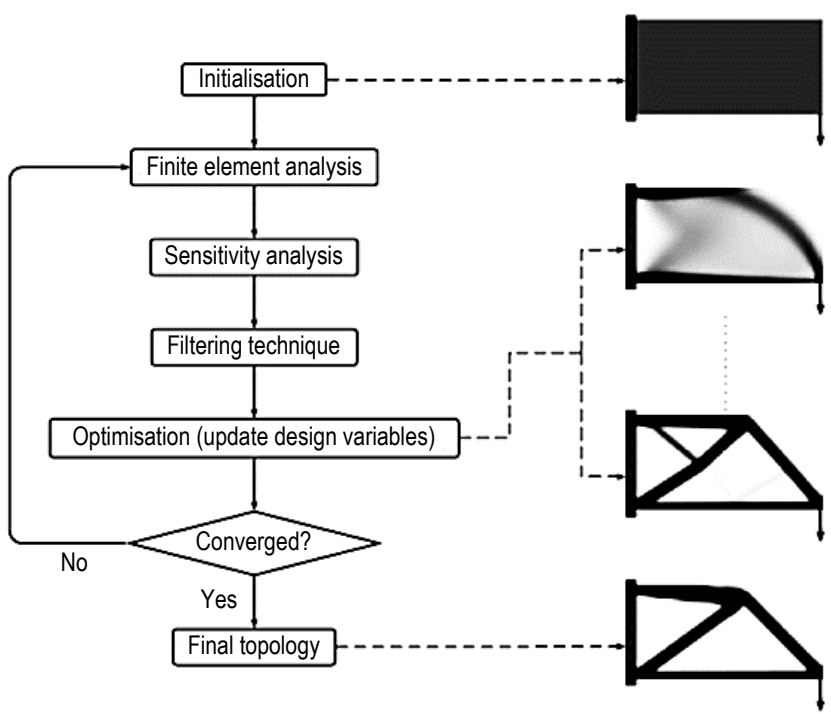

Fig. 3 Steps for topology optimisation [15-16]

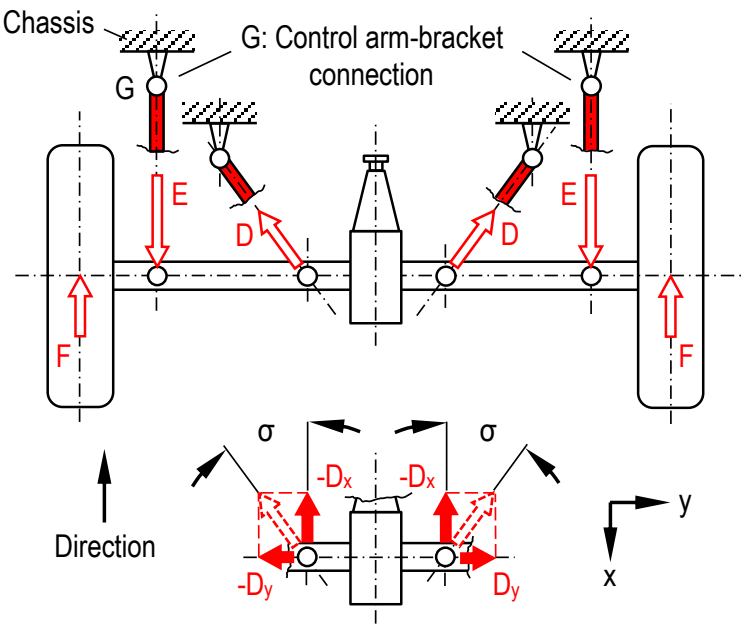

Fig. 4 Control arm forces for the longitudinal motion [17]

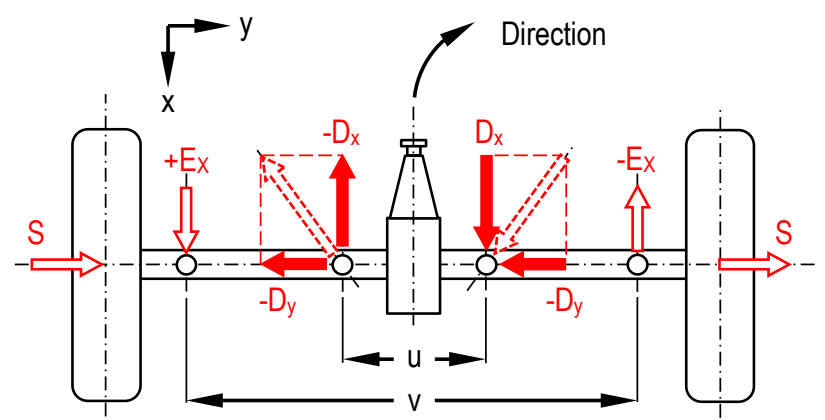

Fig. 5 Control arm forces for the turning motion [17]

\subsection{Multibody dynamics modelling}

The design loads that act on the bracket in certain service conditions were specified through an MBD model. During the MBD simulations, different load cases such as acceleration and deceleration conditions in straight or curving roads or jumping over a bump scenario were taken into consideration. The purpose of this model is to determine the maximum design load for the bracket, in whose light the mechanical design and optimisation process will be carried out by means of an FE analysis. In this model, structural elements of the vehicle were assumed to be rigid. MBD models of the rear axle and the tractor-trailer units of the articulated vehicle are seen in Figs. 6 and 7, respectively.
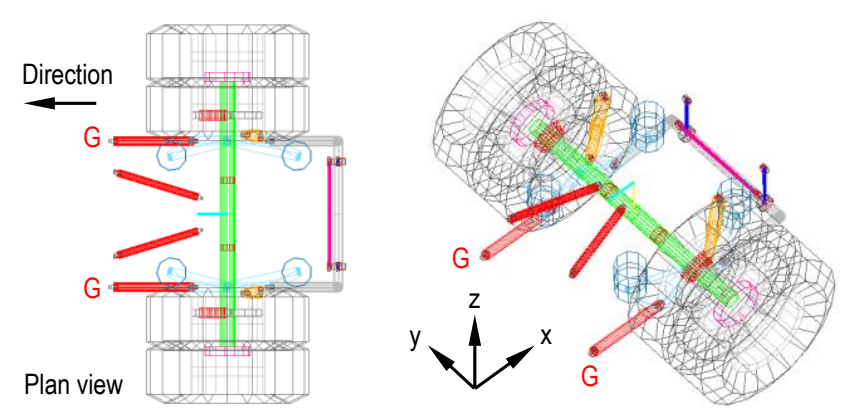

Fig. 6 MBD model of the four-link rear axle

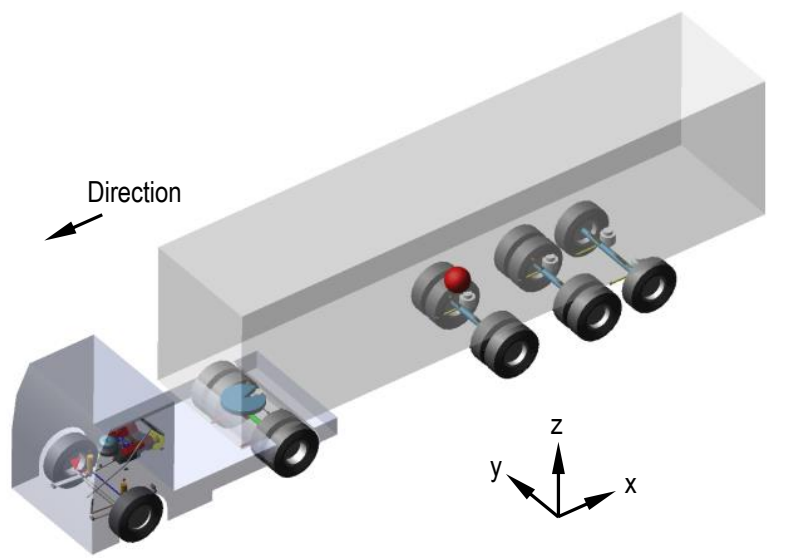

Fig. 7 MBD model of the fully articulated vehicle

The determination of the design load on the bracket was achieved by applying the selected driving manoeuvres and driving simulations to the model in Adams/Car ${ }^{\mathrm{TM}}$ environment. Correspondingly, each case was evaluated and among them, the most critical load case was determined as braking at a longitudinal deceleration value of $0.55 \mathrm{G}$. The bracket design should ensure strength to overcome such a load. The deceleration characteristic of the tractor supplied from this simulation is given in Fig. 8.

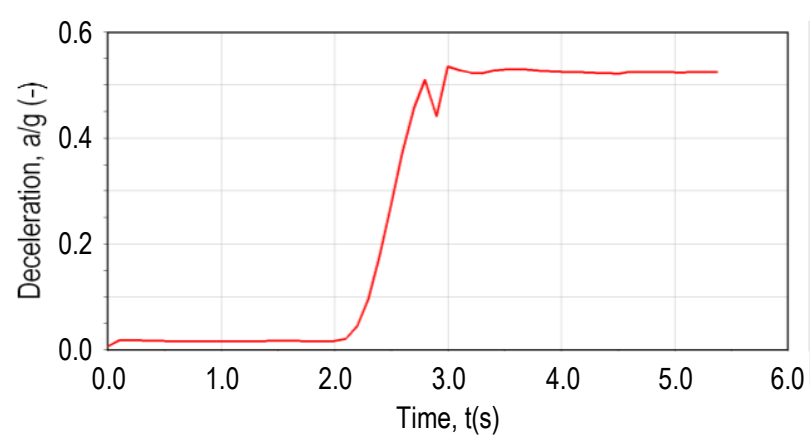

Fig. 8 Deceleration characteristic of the vehicle

\section{Initial modelling and material selection}

Throughout the sizing of the connection bracket, its relation with other components should be taken into account. The connection bracket ensures contact between the chassis and the lower control arm of the four-link rear axle. Therefore, the dimensions of the connection bracket are determined according to the settlement of lower control arm, 
since the position of chassis does not change during the positioning of axle components. The settlement of lower control arms, on the other hand, has an important role in terms of kinematic stability since they decide the position of roll centre with the upper control arms. The roll centres of the front and rear axles compose a roll axis, about which the vehicle is assumed to lean to sides during a turning motion. This effect is created by the roll moment, whose moment arm is the vertical distance between the mass centre and the roll axis on the front view of the vehicle. The roll centres and roll axis should be known in order to determine the roll behaviour of the vehicle and calculate the stiffness of the anti-roll bar properly. The roll centre position according to the arrangement of control arms is shown in Fig. 9. As a result of the length and settlement of lower control arms, the length $K$ of the connection bracket was determined. The thickness of the bracket was also decided by taking the required perpendicular distance between the chassis and lower control arm into account. The basic dimensions of the connection bracket were given in Fig. 10.

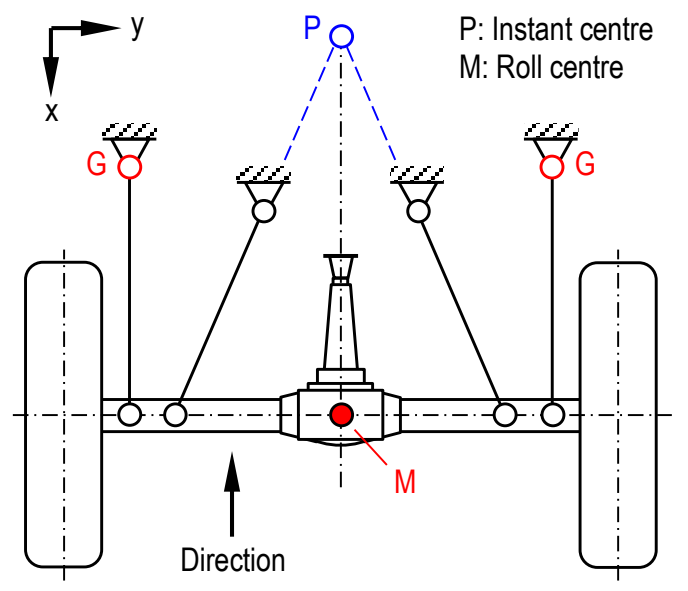

a

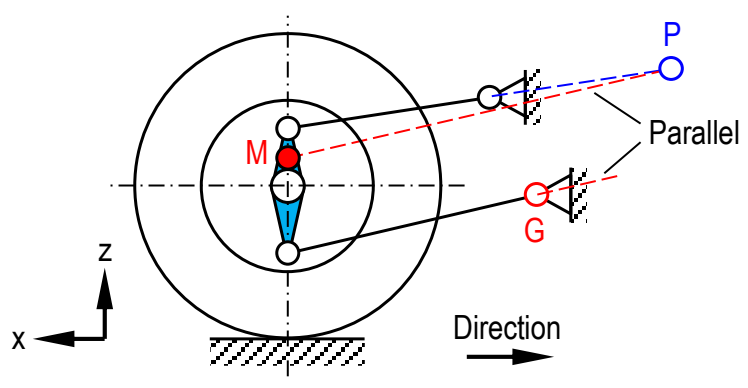

b

Fig. 9 Roll geometry of the rear axle: a - plan view; b - side view [18]

Another concern while creating a draft model of the connection bracket is the volume and configuration of the neighbour structural elements of the rear axle. Accordingly, a design volume for the geometry was determined, which should not penetrate with other elements in any position of the elements during the service. Then, the spatial positions of the connection points were determined with the chassis and the control arm, and the connection bolt holes were drilled. The structural elements of the rear axle, namely, the lower control arm 7, spring support 8 and the chassis 4 and the connection bracket 6 are given in Fig. 11 .
Ductile cast iron is used for the bracket material, whose yield strength is given as $\sigma y=420 \mathrm{MPa}$.

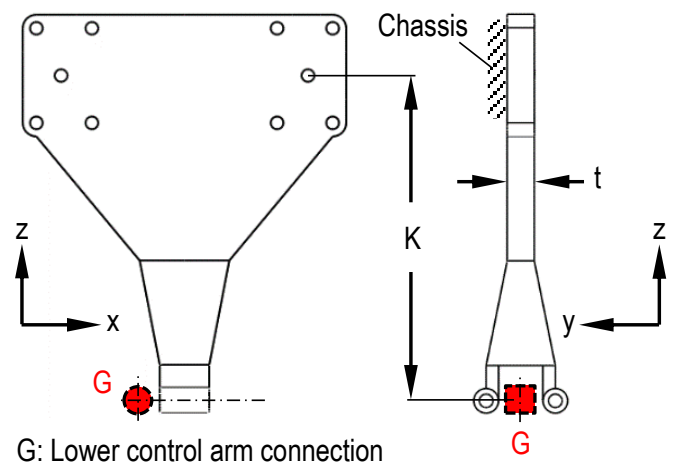

Fig. 10 Dimensions of the initial bracket design

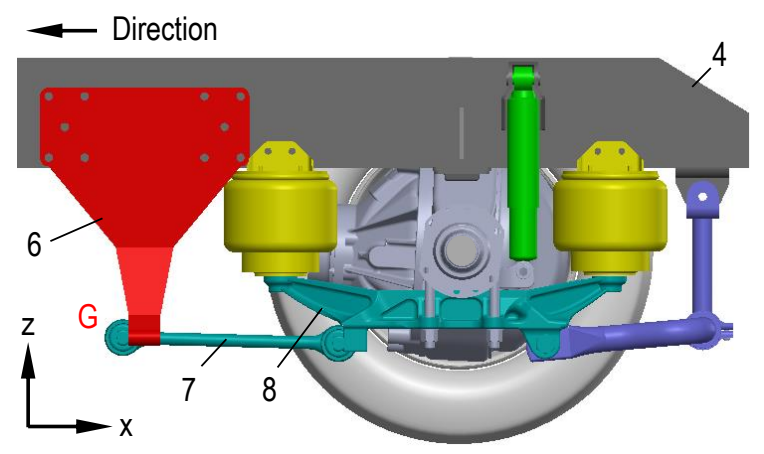

Fig. 11 Configuration of the rear axle structural elements

\section{Results}

\subsection{Lightweight design}

During the weight reduction process, FE analysis is required to carry out topology optimisation. Therefore, the initial design of bracket was subjected to a static structural analysis, which performed in ANSYS ${ }^{\circledR}$ Workbench 18.2 software environment. In this process, the lower control arm was considered as a two-force member, hence the design load was applied in control arm through the control arm axis. Once the FE analysis took place, the static structural module was connected to a topology optimisation module. For the design domain, the component body was introduced to the software. Afterwards, the exclusion regions were determined in accordance with the constructive requirements, namely, the bolt holes of connection zones of the bracket with other components and the geometry fixtures. These regions were then subtracted from the design domain, in other words, they were not included in the optimisation process. Another constraint of the topology optimisation is the "pull-out direction", which specifies the mould removal direction for the cast parts. In this design, the $y$-axis was assigned as the pull-out direction, which is indicated in Fig. 12. The mass reduction was set to 0.25 , which covers $25 \%$ of the overall structure mass. The topology optimisation result revealed that the most rigid geometry is obtained at $30 \%$ of the total mass when the control arm-bracket connection has an I-section, which can clearly be seen in Fig. 10. Subsequently, a primary producible design model was created with the influence of topology optimisation result. The topology optimisation result and the model geometry are compared in Fig. 13. Nevertheless, the 
mechanical strength of the lightened producible model should be verified by means of the following stress analysis. Equivalent stress value can be evaluated for the stress assessment since the bracket material is ductile cast iron [19-21].
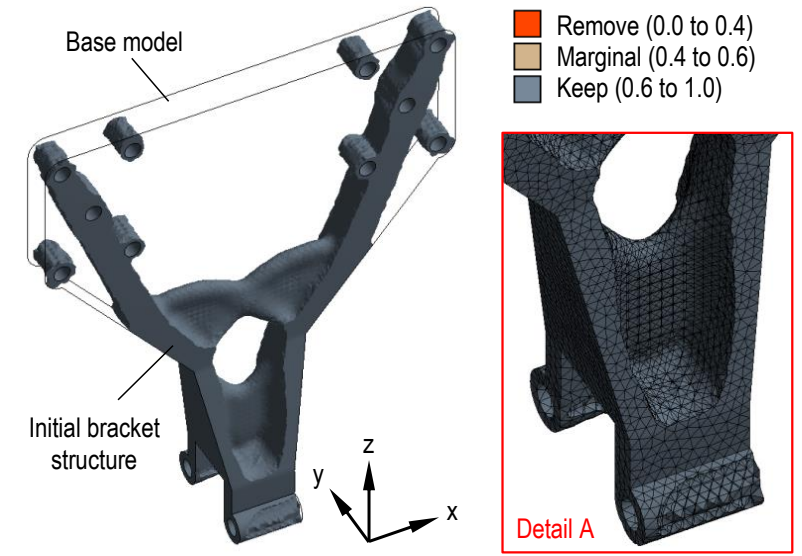

Fig. 12 Initial bracket structure obtained from the topology optimisation

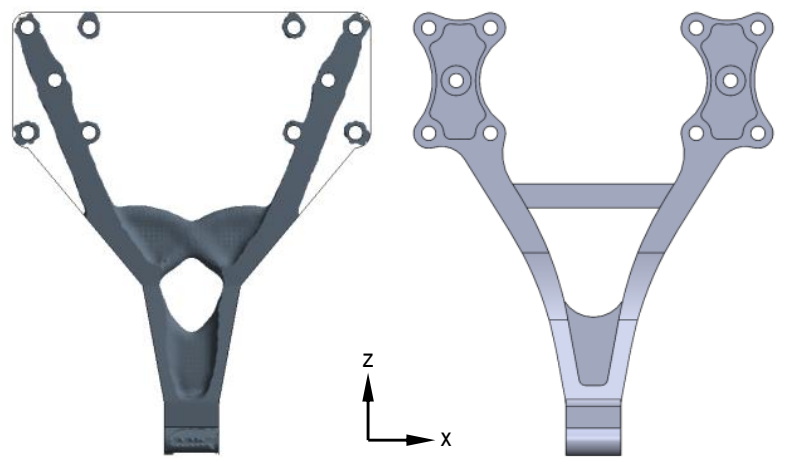

Fig. 13 Comparison of the topology optimisation result (left) and the primary producible design (right)

For the mechanical adequateness criteria, a factor of safety value of $n_{s}=1.5$ was taken into consideration [22]. Correspondingly, six regions were observed, where stress concentrations and a drop in factor of safety occurred. The stress distribution on primary design geometry was given in Fig. 14.
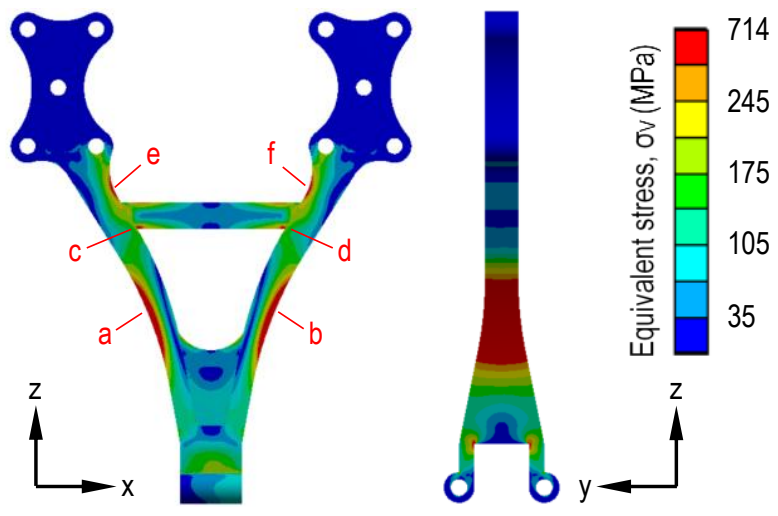

Fig. 14 Equivalent stress distribution on primary bracket design

\subsection{Stress-based design optimisation}

A stiffer design of bracket, which was obtained via topology optimisation with a higher maximum stress of the design due to more effective use of the structural domain. Consequently, after the design is reproduced in a manufacturable way, it should be subjected to the following FE analysis under the same design conditions and the following optimisation should be utilised to meet the design requirements if necessary. Hence, an FE analysis was performed with the producible design, and six critical regions (a to f) were observed, where safety factor remained below 1.5 due to the stress concentrations. The maximum von Mises stress value was monitored as $\sigma_{V \max }=714 \mathrm{MPa}$, therefore a design optimisation study was subsequently applied. Note that, only regions $\mathrm{a}-\mathrm{b}$ were observed and regions $\mathrm{c}$ to $\mathrm{f}$ were neglected, since these regions may have higher stress values due to the singularity, which causes misleading results.

During the optimisation stage, RSM (Response Surface Methodology), which is one of the most popular practices of DOE (Design of Experiments) methodology was employed through the DesignXplorer ${ }^{\mathrm{TM}}$ module of ANSYS/Workbench ${ }^{\mathrm{TM}}$. DOE-RSM is a numerical method, which is a convenient tool for parametric optimisation applications. The method correlates relations between geometric parameters of a structure and the system response to the variables, such as maximum stress or deformation under defined conditions. According to the number of input parameters, the DOE method defines a certain amount of design points, in which the input parameters take specific values. Afterwards, FE analysis is repeated at these points, and the requested system response is determined. Finally, the RSM is employed to create a continuous function of output parameters, in terms of geometric input parameters, and a surface is generated to illustrate the function of the system response. Fig. 15 illustrates the methodology.

The RSM uses a polynomial type regression model [23], which can be expressed as [24]:

$$
y=\beta_{0}+\sum_{i=1}^{k} \beta_{i} x_{i}+\sum_{i \leq j}^{k} \beta_{i j} x_{i} x_{j}+\varepsilon .
$$

When the model is written in matrix form, it becomes:

$$
\boldsymbol{y}=\boldsymbol{X \beta}+\varepsilon .
$$

In these expressions, $y$ denotes the observation vector, and $x$ represents the model matrix. $\beta$ composes of the partial regression coefficients, while $\varepsilon$ indicates the random errors. The minimisation of $\varepsilon$ yields the $\beta$ values, which are given by [25]:

$$
\hat{\boldsymbol{\beta}}=\left(\boldsymbol{X}^{T} \boldsymbol{X}\right)^{-1} \boldsymbol{X}^{T} \boldsymbol{y}
$$

The design points were determined with "Central Composite Design" type data distribution. In CCD, a combination of a two-level factorial or fraction and $2 k$ number of either axial or star points is employed. Therefore, the total number of runs is determined by means of the formula $2^{k}+2 k+n_{c}$ that are taken advantage of during the determination of design points, while $n_{c}$ represents the total number of centre runs.

During the design optimisation of the bracket, two geometric input parameters, $L$ and $h$, were chosen as design variables (or factors). In accordance with the upper and 
lower limits of these variables, design points were specified, in which FE analysis is updated, and the response surface is generated. The dimensions $L$ and $h$ are shown in Fig. 16, which denote the vertical position and the cross-sectional height of the reinforcement rib, respectively. The values of the variation range of these factors are given in Table 1.

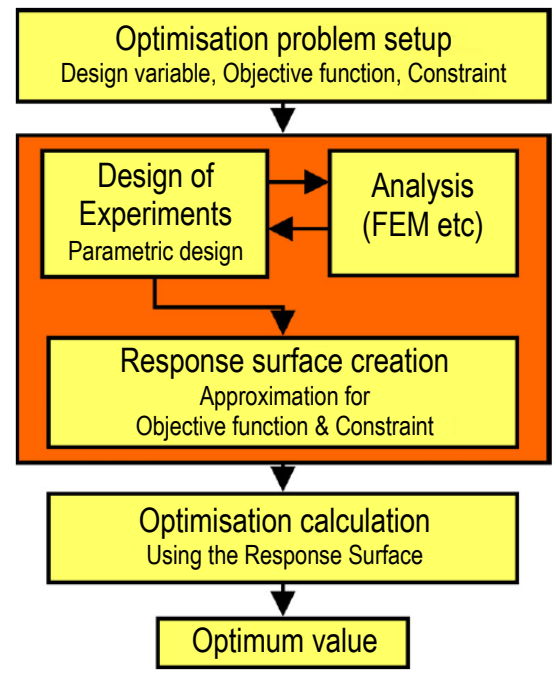

$\mathrm{a}$

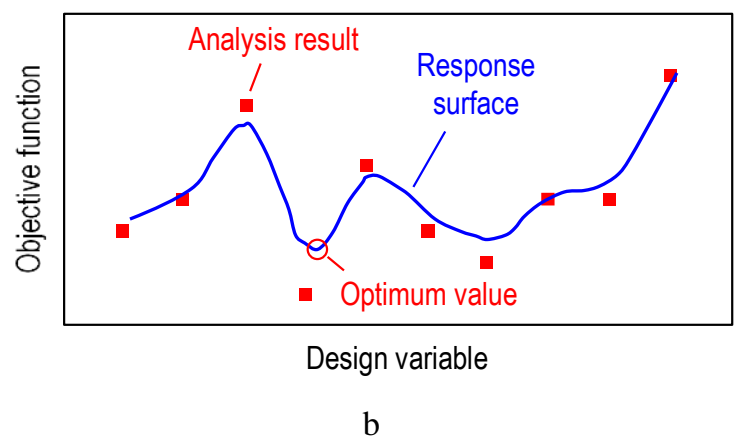

Fig. 15 Response surface methodology (RSM): a - [26]; $\mathrm{b}$ - schematic for the response surface [27]

Table 1

Variation range of the design factors

\begin{tabular}{|l|l|l|}
\hline Factor & Min. value, $\mathrm{mm}$ & Max. value, $\mathrm{mm}$ \\
\hline$L$ & 200 & 350 \\
\hline$h$ & 25 & 45 \\
\hline
\end{tabular}

Correspondingly, the optimisation module specified nine design points (Table 2), which consist of the combination of numerous values of parameters $L$ and $h$. Stress values on the structure at these points were hereafter determined via the FE analyses. The maximum von Mises stress value was revealed as a function of these parameters. The function benefited the response surface, which bases on the $\sigma_{\mathrm{V}}$ values at design points. The design points were generated by the software according to CCD. An illustration of the 3D and $2 \mathrm{D}$ view of the response surface is given in Figs. 16 and 17, respectively. Subsequently, GDO (Goal Driven Optimisation) method was utilised to determine an optimal value among the combinations of design variables that satisfies the minimum stress concentration on the design, regarding the optimisation goals and constraints. In this design, a design restriction of maximum allowable deformation value was specified as $\delta_{\max } \leq 2 \mathrm{~mm}$ which is the maximum desired deformation value for the most critical load case.
Table 2

Design points

\begin{tabular}{|l|l|l|}
\hline Sample No. & $h, \mathrm{~mm}$ & $L, \mathrm{~mm}$ \\
\hline 1 & 35 & 275 \\
\hline 2 & 25 & 275 \\
\hline 3 & 45 & 275 \\
\hline 4 & 35 & 200 \\
\hline 5 & 35 & 350 \\
\hline 6 & 25 & 200 \\
\hline 7 & 45 & 200 \\
\hline 8 & 25 & 350 \\
\hline 9 & 45 & 350 \\
\hline
\end{tabular}
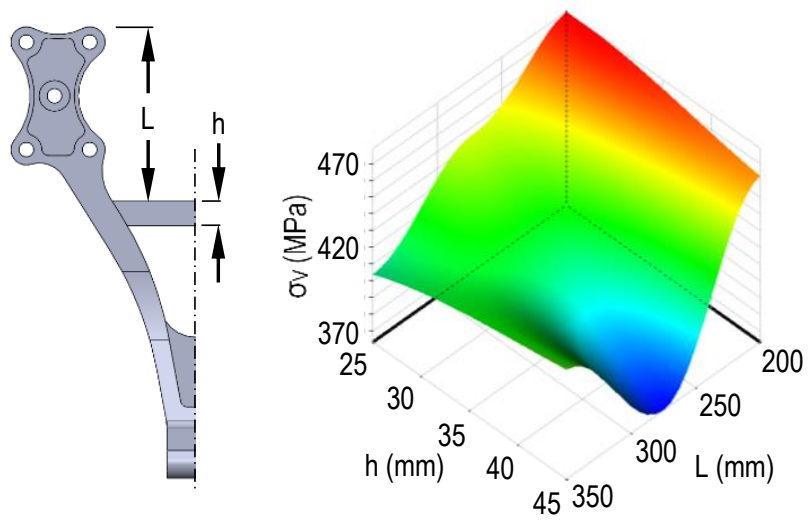

Fig. 16 Response surface for equivalent stress
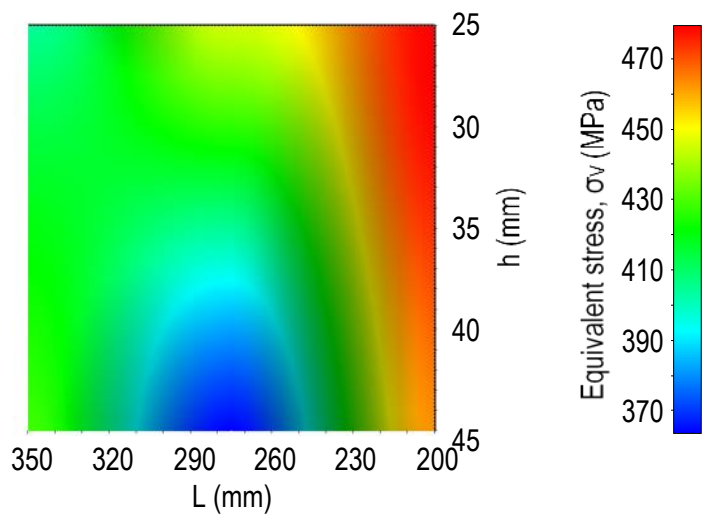

Fig. 17 Response surface for equivalent stress

A CAD model for the bracket with the optimised design parameters was afterwards generated and subjected to an FE analysis. The observations on the critical regions indicated that a decrease in maximum stress values on the majority of these regions was monitored. The overall distribution of $\sigma_{V}$ on the design with optimised parameters is shown in Fig. 18.

\subsection{Design enhancement and finalisation}

Eventually, a final improvement was implemented to the optimised design, which consists of the edge fillets in order to achieve smooth surfaces between the transition regions and prevent the stress concentrations on the component. The improved model with fillets is shown in Fig. 19. The radiuses and ribs that were added to the design also by taking the result of topology optimisation into account, which is given in Fig. 12. An FE analysis of revised optimal design was carried out in order to observe the effects of final improvements to the model. 


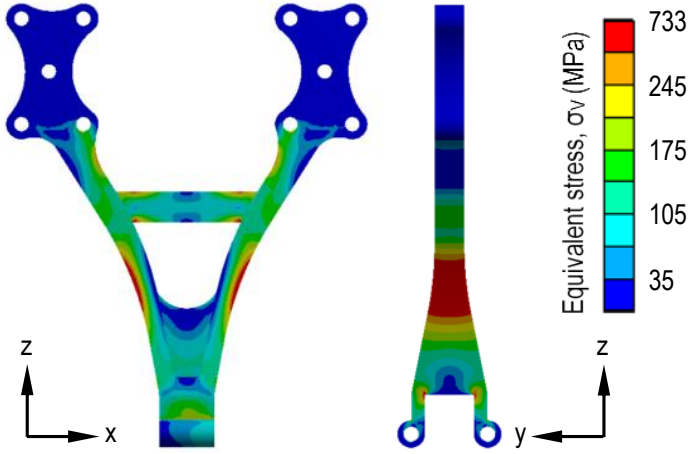

Fig. 18 Stress distribution on the optimised bracket design

According to the analysis result, it was seen that the design requirements of the bracket were satisfied for the critical load case. The stress distribution of static analysis is shown in Fig. 20.
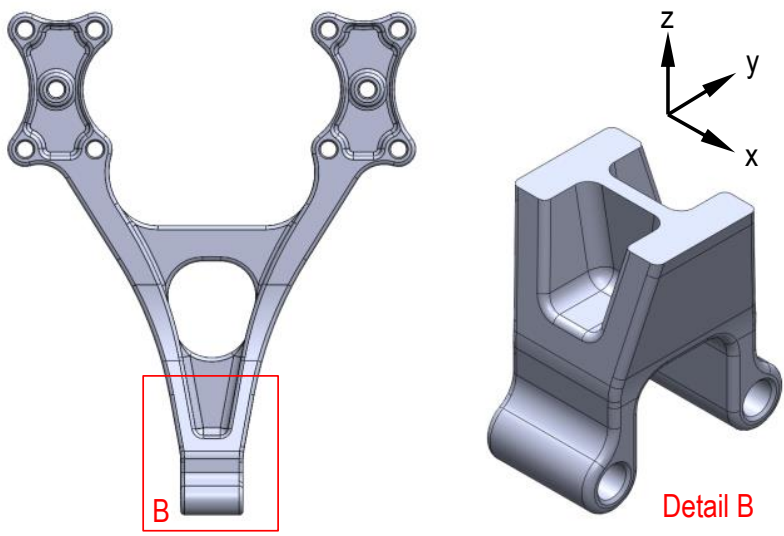

Fig. 19 Final design with edge fillets
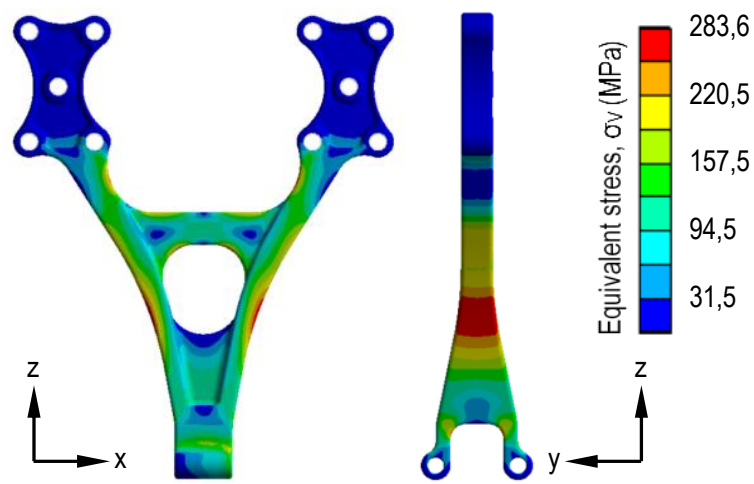

Fig. 20 Stress distribution on the final design

\section{Discussion}

The change in the maximum von-Mises stress values on regions a to $f$ were analysed and compared for the primary, optimised and revised bracket models. The maximum stress value change in these regions are illustrated in the diagram at Fig. 21. The FE analyses of different bracket designs reveal that the RSM optimisation study has a significant effect at the critical regions a, b, e and f. Nonetheless, at the regions $\mathrm{c}$ and $\mathrm{d}$, maximum $\sigma_{\mathrm{V}}$ values were still higher than the yield strength of the material after the design study, owing to the sharp edges and sudden transition regions. The effect of the additional edge fillets to the design optimisation result was a $63 \%$ decrease in stress values at regions $\mathrm{c}$ and d, which is also pointed out in Fig. 21 .

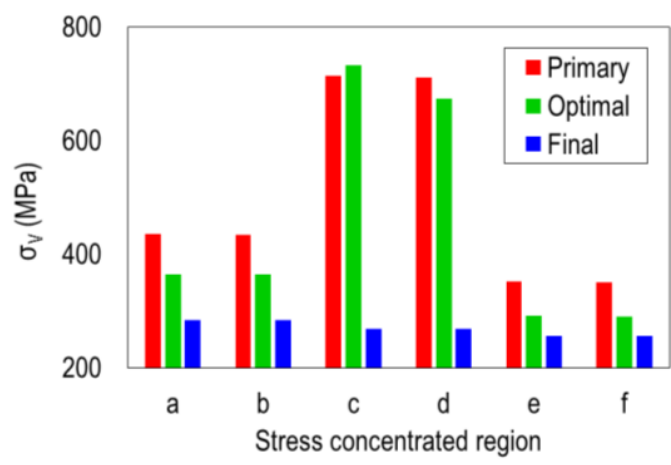

Fig. 21 Comparison of the equivalents stress concentration at the critical regions of the bracket designs

The factor of safety for the final design of the bracket was reasonable and observed as $n_{S}=1.48$. The directional deformation (in $\mathrm{x}$-axis) values of the connection between the lower arm and bracket were also analysed and the design target of deformation for all three design samples was reached. Directional deformation values for three different designs were given in Fig. 22.

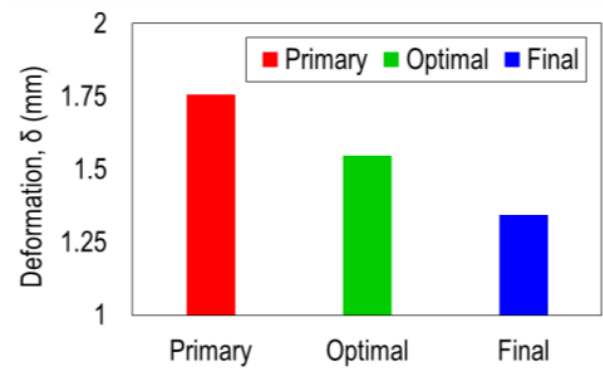

Fig. 22 Deformations of the lower control arm connection of the bracket region for the bracket designs

The results, which are also indicated in the figure reveal that the deformation value is reduced by about $12 \%$ compared with the primary design by the size optimisation. Furthermore, when the edge fillets are also taken into account, the decrease in deformation raises to $13 \%$. In practice, such deformation drop effects may be neglected. Fig. 23 illustrates the component mass comparison of base, primary, optimal and final bracket designs. As it is also pointed out in the figure that a negligible increase in mass (lower than $1 \%$ ) compared with the primary producible design occurred due to the results of RSM-based design study. However, the mass of the final design is $63 \%$ lighter rather than the initial design, which can be seen in Fig. 23.

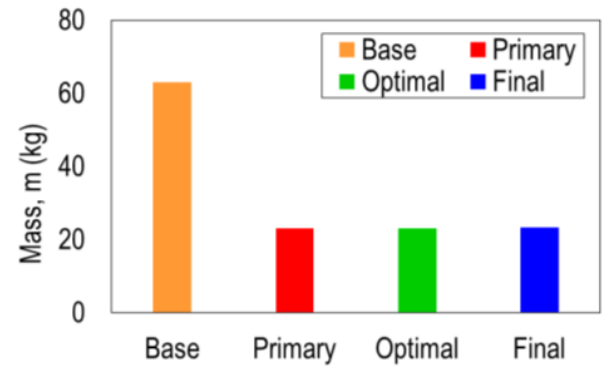

Fig. 23 Mass comparison for the bracket designs

\section{Conclusion}

In the scope of this study, a numerical case study was performed for the lightweight design of a connection 
bracket, which is used in the solid rear axle of an articulated truck.

The design process consists of a composite method including different techniques, namely, topology optimisation, finite element (FE) analysis and response surface methodology (RSM)-based size optimisation. During the topology optimisation phase, the stiffest design for the connection bracket was specified. Then, the bracket was redesigned in a producible way according to the resulting form. Subsequently, the new design for the connection bracket was subjected to an FE analysis to determine the critical stress regions, which result in an insufficient factor of safety value. In the final stage, the critical stress values were reduced through the RSM-based size optimisation. Numerical results indicated that, by using topology optimisation, it is possible to reduce the mass of the bracket about $63 \%$ in comparison with the base design. FE-based loading simulations also showed that equivalent stress at the most critical regions of the component was decreased up to $62 \%$. According to these results, final design satisfies the strength and stiffness conditions for critical load case.

\section{Acknowledgement}

Authors acknowledge the licensed software support of BMC Otomotiv ve Sanayi Ticaret A.Ş. in Izmir, Turkey.

\section{References}

1. Reimpell, J.; Stoll, H.; Betzler, J. W. 2001. The Automotive Chassis: Engineering Principles. 2nd edition. Warrendale: Society of Automotive Engineers, Inc., $444 \mathrm{p}$.

2. Dixon, J. 2009. Suspension Geometry and Computation. 1st edition. Chicester, West Sussex: John Wiley \& Sons, $417 \mathrm{p}$. https://doi.org/10.1002/9780470682906.

3. Bendsoe, M. P.; Kikuchi, N. 1988. Generating optimal topologies in structural design using a homogenization method, Comp. Meth. Appl. Mech. Eng. 71: 197-224. https://doi.org/10.1016/0045-7825(88)90086-2.

4. Yang, R. J.; Chahande, A. I. 1995. Automotive applications of topology optimization, Structural and Multidisciplinary Optimization 9(3-4): 245-249. https://doi.org/10.1007/BF01743977.

5. Mayer, R. R.; Kikuchi, N.; Scott, R. A. 1996. Application of Topological Optimization Techniques to Structural Crashworthiness, International Journal for Numerical Methods in Engineering 39: 1383-1403. https://doi.org/10.1007/BF01743977.

6. Bendsoe, M. P.; Sigmund, O. 1999. Material Interpolation Schemes in Topology Optimization, Archive of Applied Mechanics 69(9-10): 635-654. https://doi.org/10.1007/s004190050248.

7. Bendsøe, M. P.; Sigmund, O. 2003. Topology Optimization, Theory, Methods, and Applications. Berlin: Springer, $370 \mathrm{p}$. https://doi.org/10.1007/978-3-662-05086-6.

8. Hara K.; Zha, H.; Haegawa, T. 1999. Topology-adaptive modeling of objects by a level set method with multi-level stopping conditions, Proceedings 1999 International Conference on Image Processing 4: 371-375. https://doi.org/10.1109/ICIP.1999.819616.
9. Wang, M. Y.; Wang, X.; Guo, D. 2003. A level set method for structural topology optimization, Computer Methods in Applied Mechanics and Engineering 192(12): 227-246. https://doi.org/10.1016/S0045-7825(02)00559-5.

10. Fredricson, H.; Johansen, T.; Klarbing, A.; Petersson, J. 2003. Topology Optimization of Frame Structures with Flexible Joints, Structural and Multidisciplinary Optimization 25(3): 199-214. https://doi.org/10.1007/s00158-003-0281-z.

11. Jang, G. W.; Yoon, M. S.; Park, J. H. 2010. Lightweight flatbed trailer design by topology and thickness optimization, Structural and Multidisciplinary Optimization 41(2): 295-307. https://doi.org/10.1007/s00158-009-0409-x

12. Wang, J.; Wang, T.; Jang, Y.; Peng, Z.; Li, Z.; Wang, N. 2012. Topology Optimization Design of a Heavy Truck Frame, Proceedings of the FISITA 2012 World Automotive Congress 195: 219-227. https://doi.org/10.1007/978-3-642-33835-9_21.

13. Tang, L.; Wu, J.; Liu, J.; Jiang, C.; Shangguan, W. B. 2015. Topology Optimization and Performance Calculation for Control Arms of a Suspension, Advances in Mechanical Engineering 6: 1-10. https://doi.org/10.1155/2014/734568.

14. Nuțu, E. 2015. Interpretation of parameters in strain energy density bone adaptation equation when applied to topology optimization of inert structures, Mechanics 21 (6): 443-449. https://doi.org/10.5755/j01.mech.21.6.12106.

15. Johnsen, S. 2013. Structural Topology Optimization: Basic Theory, Methods and Applications, Norwegian University of Science and Technology, M. Eng. Thesis, Trondheim: $179 \mathrm{p}$.

16. Wang, S. 2007. Krylov Subspace Methods for Topology Optimization on Adaptive Meshes. University of Illinois, PhD Thesis, Illinois: $124 \mathrm{p}$.

17. Reimpell, J. 1973. Fahrwerktechnik, Bd. 2. Würzburg: Vogel-Verlag, 352 p (in German).

18. Estorff, H. E. 1969. Technische Daten Fahrzeugfedern Teil 3: Stabilisatoren. Werdohl: Stahlwerke Südwestfalen AG, 146 p. (in German).

19. Kim, D. J.; Lee, Y. M.; Park, J. S.; Seok, C. S. 2008. Thermal stress analysis for a disk brake of railway vehicles with consideration of the pressure distribution on a frictional surface, Materials Science and Engineering 483-484: 456-459. https://doi.org/10.1016/j.msea.2007.01.170.

20. Bagnoli, F.; Dolce, F.; Bernabei, M. 2009. Thermal fatigue cracks of fire fighting vehicles grey iron brake discs, Engineering Failure Analysis 16: 152-163. https://doi.org/10.1016/j.engfailanal.2008.01.009.

21. Park, S.; Lee, J.; Moon, U.; Kim, D. 2010. Failure Analysis of a Planetary Gear Carrier of 1200HP Transmission, Engineering Failure Analysis 17(2): 521-529. https://doi.org/10.1016/j.engfailanal.2009.10.001.

22. Roloff, H.; Matek, W. 1972. Maschinenelemente. 5th edition. Braunschweig: Vieweg \& Sohn GmbH, 575 p. (in German).

23. Park, K.; Heo, S. J.; Kang, D. O.; Jeong, J. I.; Yi, J. H.; Lee, J. H.; Kim, K. W. 2013. Robust design optimization of suspension system considering steering pull reduction, International Journal of Automotive Technology 14: 927-933. 
https://doi.org/10.1007/s12239-013-0102-3.

24. Myers, R. H.; Montgomery, D. C.; Anderson-Cook, C. M. 2009. Response Surface Methodology, Process and Product Optimization Using Design of Experiments. 3rd edition. New Jersey: John Wiley \& Sons, 704 p.

25. Han, H.; Park, T. 2004. Robust optimal design of multi-body systems, Multibody System Dynamics 11: $167-183$

https://doi.org/10.1023/B:MUBO.0000025414.28789.3 4.

26. Amago, T. 2002. Sizing optimization using response surface method in FOA, R\&D Review of Toyota CRDL 37 (1): 1-7.

27. Amago, T. 1998. Response surface methodology and its application to automotive suspension design. [online] Toyota Central Research Laboratories, Nagoya, Japan [accessed 09 September 2019] Available from Internet: http://www-personal.umich.edu/ kikuchi/Research/rsm_amago.pdf.

M. M. Topaç, M. Karaca, B. Aksoy, U. Deryal, L. Bilal

\section{LIGHTWEIGHT DESIGN OF A REAR AXLE CONNECTION BRACKET FOR A HEAVY COMMERCIAL VEHICLE BY USING TOPOLOGY OPTIMISATION: A CASE STUDY}

S u m m a r y

An important design challenge of modern vehicles is mass reduction. Hence in many cases, mechanical design of vehicle components covers different optimization processes. One important structural optimization technique which is highly utilised in weight reduction applications is the topology optimization. This paper contains a multi-stage optimization based on the topology and design optimizations. During this study, the mechanical design of a rear axle-chassis connection bracket is achieved. First of all, the design load of the bracket was determined through a multibody dynamics analysis. This load case was determined among various driving conditions and the most critical load case was indicated as the design load of the bracket. This process was executed by using Adams/Car ${ }^{\mathrm{TM}}$ software. Subsequently, a design volume for the bracket was decided, which specifies the domain of topology optimization that will be employed later on. The determination of the design domain was made by considering the structural position of the design component, the neighbor components of the rear axle and the chassis. In this manner, the basic shape and dimensions of the bracket were created. The unnecessary volume of the draft design, which is not properly loaded under the design conditions was determined and removed from the design by means of topology optimization. The topology optimization was run in topology optimization module of ANSYS ${ }^{\circledR}$ Workbench 18.2 finite element analysis (FEA) software package. In the light of the primary shape obtained from the topology optimization study, a producible initial design model was built. This model was then subjected to FE analysis under the same circumstances with the draft model, in order to perform strength and deformation assessments of the initial design. Correspondingly, the critical regions were determined where stress concentrations were observed. The model was updated in a way that the stress values were reduced in these regions through the response surface methodology (RSM). The comparisons between the result and the initial geometries reveal that the mass of the connection bracket was reduced by $63 \%$. Besides, the total deformation which was dropped by the design optimization is $13 \%$ lower than the initial design that was generated with the influence of topology optimization result.

Keywords: lightweight design, mechanical design, multibody dynamics (MBD), topology optimisation, finite element analysis (FEA), response surface methodology (RSM), heavy commercial vehicle.

Received April 09, 2019

Accepted February 03, 2020 